\title{
Clinical determinants of adrenal vein sampling success
}

\author{
Maxime Berney ${ }^{a}$, Marc Maillarda, Francesco Doenz ${ }^{b}$, Maurice Matter ${ }^{c}$, Antoinette Pechère-Bertschid, \\ Michel Burniera, Gregoire Wuerzner ${ }^{a}$
}

a Service of Nephrology and Hypertension, Lausanne University Hospital, Switzerland

b Service of Radiology, Lausanne University Hospital, Switzerland

c Service of Visceral Surgery, Lausanne University Hospital, Switzerland

d Medical Policlinic and Division of Endocrinology, Geneva University Hospital, Switzerland

\section{Summary}

Background: Primary aldosteronism (PA) is one of the most prevalent forms of secondary hypertension, which may be cured by unilateral adrenalectomy. Adrenal vein sampling (AVS) is considered the gold-standard procedure for distinction between bilateral and unilateral aldosterone hypersecretion. However, the procedure is viewed as a technical challenge. The objectives of this study were to define the success rate of AVS, and its clinical determinants, and to compare the accuracy of high-resolution adrenal computed tomography (CT) with AVS.

Methods: In this single-centre retrospective study, patients with biologically proven PA who were referred for AVS between 2009 and 2014 were included. Adrenal vein catheterisation was considered selective if the selectivity index (adrenal vein / inferior vena cava cortisol) was $\geq 2$.

Results: Data from 68 patients (48\% women) were available. The success rate of catheterisation in bilateral AVS was $60 \%$ (41/68). The significant clinical determinants of success were male sex $(r=0.35, p=0.004)$, a higher body mass index (BMI) $(r=0.54, p=0.001)$ and plasma creatinine $(r=0.25, p=0.048)$ in univariate analysis. In multivariate linear regression analysis, only BMI was associated with success (coefficient $\beta=0.049$, $p=0.004)$. CT was discordant with AVS in $53 \%$ of patients, and would have resulted in inappropriate adrenalectomy in $43 \%$ of the patients and inappropriate exclusion from surgery in $10 \%$ of the cases.

Conclusion: Clinical characteristics such as BMI and sex may influence the success rate of AVS. The inaccuracy of CT may result in inappropriate treatment proposals if the indication for surgical intervention is based on CT only.

\section{Background}

Primary aldosteronism (PA) is one of the commonest forms of secondary hypertension. Its prevalence in hypertensives patients is $10 \%$ and may rise to $20 \%$ in selected patients with resistant hypertension [1, 2]. In this selected population, PA may account for one third of all cases of secondary hypertension [3]. Among sub-

types of PA, aldosterone-producing adenoma (APA) and bilateral adrenal hyperplasia (BAH) are the two most prevalent subtypes and are responsible for $>95 \%$ of all PA [1]. Interestingly, the prevalence of APA compared with $\mathrm{BAH}$ is higher in centres where adrenal vein sampling is available [1]. Early identification and specific treatment of patients with PA is important because these patients carry an increased risk of target organ damage and an increased risk of cardiorenal complications when compared with essential hypertension patients with similar blood pressure levels [4]. Finally, most patients with PA and unilateral aldosterone oversecretion benefit from a surgical approach, resulting in normalisation of aldosterone secretion or blood pressure, better blood pressure control with less drugs and a regression of target organ damage [5-8]. The gold standard method for identifying a unilateral overproduction of aldosterone is through adrenal vein sampling (AVS) [9]. AVS consists of direct assessment of hormonal secretion in the adrenal veins and inferior vena cava. Although AVS appears to be a straightforward technique, it is underused worldwide owing to the belief that it is technically challenging, invasive, associated with risks and not always necessary $[9,10]$. As no data regarding clinical determinants of AVS success have been published, the purpose of this study was to define the success rate of AVS and to identify its clinical determinants, as well as to compare the accuracy of high-resolution adrenal computed tomography (CT) with AVS in a referral centre in Switzerland.

\section{Methods}

This study was a retrospective, single-centre-based analysis of all patients with PA who were referred to the Lausanne University Hospital for AVS between 2009 and 2014. AVS was proposed for all patients with PA in whom adrenalectomy was a possible therapeutic option. Antihypertensive drugs were stopped 2 weeks prior to AVS, except for spironolactone which was 
stopped 6 weeks before the intervention. The only antihypertensive drugs allowed before AVS were the alpha-blocker doxazosine or calcium channel blockers. The study was approved by the local institutional review committee.

\section{Radiological imaging}

High-resolution contrast computed tomography (CT) or magnetic resonance imaging (MRI) were used for the radiological definition of normal adrenal glands, unilateral adrenal adenoma, bilateral adrenal adenoma, unilateral adrenal hyperplasia and bilateral adrenal hyperplasia.

\section{Adrenal vein sampling}

AVS was performed under local anaesthesia by the same experienced vascular radiologist via a percutaneous right trans-femoral vein approach using a standardised protocol. The order of catheterisation and blood sampling was proximal vena cava, distal vena cava, the left adrenal vein and finally the right adrenal vein.

High-resolution CT acquisition during the procedure was used to localise the tip of the catheter precisely, allowing the distinction between the adrenal vein and a hepatic accessory vein when necessary. AVS was performed sequentially without cosyntropin stimulation.

\section{Samples}

Plasma renin activity and plasma aldosterone were measured with commercial radioimmunometric assays, (RENCTK (Angiotensin I) RIA, DiaSorin, Saluggia, Italy and ALDO RIACT ${ }^{\circledR}$ Kit, Cisbio Bioassays, Codolet, France, respectively). Serum cortisol levels were measured with FPIA (fluorescence polarisation immunoassay) on an AxSYM instrument (Abbott Diagnostics. Lake Forest, Illinois, USA).

Table 1: Baseline demographic characteristics.

\begin{tabular}{lll}
\hline & & n (found) \\
\hline Gender, male/female & $35 / 33$ & 68 \\
\hline Age, years & $47.2 \pm 12.1$ & 68 \\
\hline BMI, $\mathrm{kg} / \mathrm{m}^{2}$ & $29 \pm 5.6$ & 66 \\
\hline Potassium, $\mathrm{mmol} / \mathrm{l}$ & $3.26 \pm 0.55$ & 66 \\
\hline Creatinine, $\mathrm{mmol} / \mathrm{l}$ & $75.5 \pm 18.1$ & 63 \\
\hline Office SBP, $\mathrm{mm} \mathrm{Hg}$ & $169 \pm 28$ & 25 \\
\hline Office DBP, $\mathrm{mm} \mathrm{Hg}$ & $105 \pm 14$ & 25 \\
\hline Number of antihypertensive drugs & $2.1 \pm 1.2$ & 68 \\
\hline PRA, $\mathrm{ng} / \mathrm{ml} / \mathrm{h}$ & $0.06[0.04 ; 0.24]$ & 44 \\
\hline PAC, $\mathrm{pg} / \mathrm{ml}$ & $250[190 ; 442]$ & 39 \\
\hline
\end{tabular}

Data are presented as means \pm standard deviation, except non normally distributed data which are presented as medians an interquartiles. $\mathrm{BMI}=$ body mass index; $\mathrm{PRA}=$ plasma renin activity; $\mathrm{PAC}=$ plasma aldosterone concentration; $\mathrm{SBP}=$ systolic blood pressure; $\mathrm{DBP}=$ diastolic blood pressure

\section{Selectivity and lateralisation indexes}

AVS was considered successful if right and left adrenal venous cortisol concentration was $\geq 2$ (selectivity index; SI) times higher than the inferior vena cava plasma cortisol concentration. Lateralisation of aldosterone secretion (defined as the ratio of the higher [dominant] over the lower [nondominant] plasma aldosterone concentration / plasma cortisol concentration ratio) was considered present if the lateralisation index (LI) was $\geq 4$. These ratio indexes are recommanded by the European expert consensus statement [9].

\section{Statistic analysis}

Statistical analysis was performed using STATA $^{\circledR}$ Data Analysis and Statistical Software, version 12.1. In the descriptive analysis, the variables are presented as mean \pm standard deviation or median with interquartile range if data were not normally distributed. Comparisons between the groups were performed using $\chi^{2}$ tests for differences in the proportions of categorical variables, and two-sample Wilcoxon rank sum tests for ordinal variables and variables that were not normally distributed. Spearman rank correlation was calculated between physical characteristic and success of adrenal vein sampling. For the relationship between success rate as the dependent variable and body mass index (BMI) categories as the independent variables of interest, we used as predictors in the fully adjusted models of age, sex and plasma creatinine. We used a nonparametric test for trend across categories of BMI (nptrend function in Stata developed by Cuzick, which is an extension of the Wilcoxon rank-sum test). All p-values of $<0.05$ were considered statistically significant.

\section{Results}

Sixty-eight patients were referred for AVS between 2009 and 2014. Sixty-six of them had complete AVS and radiological imaging data. Baseline demographic characteristics are presented in table 1 . Out of all the patients, $25 \%$ had a normal serum potassium level (mean $3.26 \pm 0.55 \mathrm{mmol} / \mathrm{l}$ ). All patients had hypertension with a mean systolic blood pressure of $169 \pm 28 \mathrm{~mm} \mathrm{Hg}$ and mean diastolic blood pressure of $105 \pm 14 \mathrm{~mm} \mathrm{Hg}$. On average, patients were taking $2.2 \pm 1.2$ antihypertensive drugs. No adverse events related to AVS were reported.

\section{CT and MRI results}

The most common adrenal abnormalities detected were left adrenal nodules, in $48 \%(32 / 66)$ of the patients, followed by right adrenal nodules in $21 \%$ of the patients (14/66). Seventeen percent of the examina- 
tions (11/66) showed normal adrenals. Finally, 5\% (3/66) showed bilateral abnormalities, of which 5\% (3/66) were bilateral adenomas and $2 \%(1 / 66)$ were bilateral hyperplasia. Adrenal imaging results are summarised in figure 1.

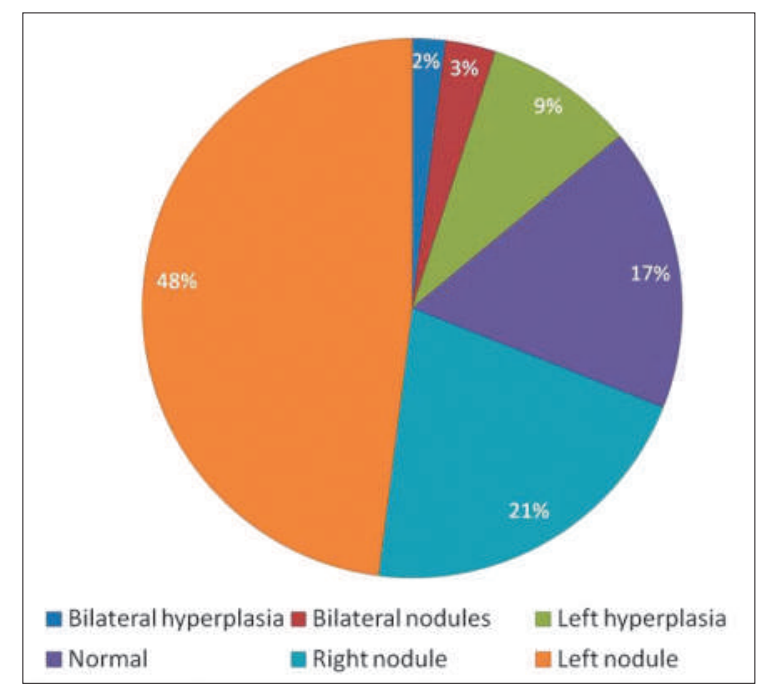

Figure 1: Radiological adrenal gland findings.

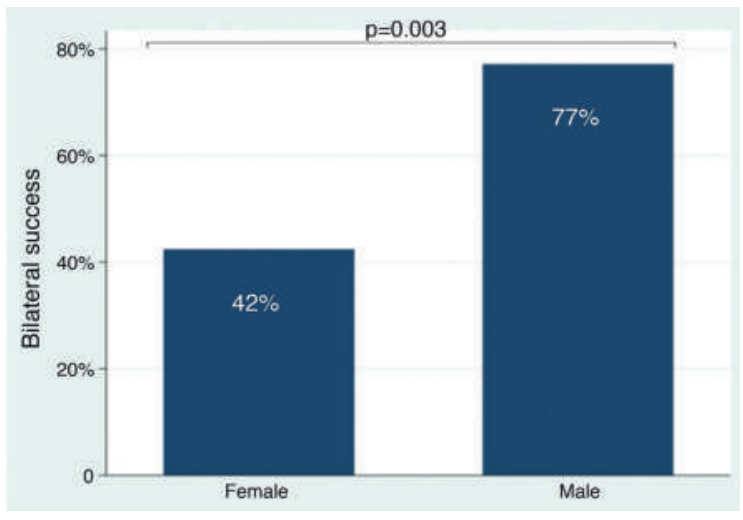

Figure 2: Rate of bilateral AVS success in women and men.

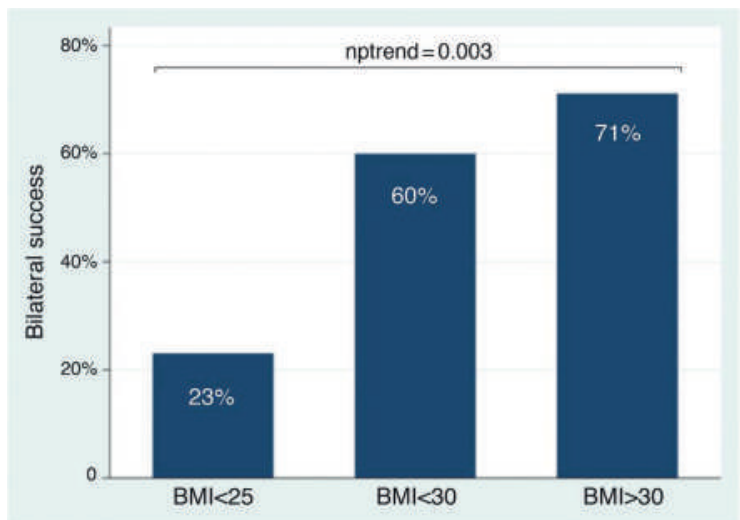

Figure 3: Rate of bilateral AVS success according to body mass index (BMI).

\section{AVS success rate and clinical determinants}

Bilateral AVS (SI $\geq 2$ in both adrenal veins) was successful in $60.3 \%$ (41/68) of patients. The success rate of left adrenal vein catheterisation tended to be higher than the right adrenal vein ( $82 \%$ vs $71 \%, \mathrm{p}=0.11)$. Male sex (fig. 2) and categories of BMI were both associated with bilateral AVS success (fig. 3). Bilateral AVS success was indeed achieved in $77 \%$ of the male population against $42 \%$ in female population. In unavariate analysis, sex, BMI and plasma creatinine were associated with catheterisation success rate (table 2). In multi-variate analysis, only a BMI was significally correlated with a high success rate, with a high BMI increasing the rate of success (table 3).

\section{Comparison between AVS and adrenal imaging} In order to compare results of AVS with the results of imaging, we use the cases of the 39 patients with bilateral AVS success. In $53 \%$ of cases, AVS results were discordant to the results of radiological imaging. The nature of the discordant results were as follows: 2 radiological images showed bilateral abnormalites but AVS concluded to unilateral aldosterone secretion, 2 radiological studies showed no abnormalities but AVS concluded to unilateral aldosterone secretion, 11 radiological studies showed unilateral abnormalites but AVS showed no lateralisation of aldosterone secretion. Finally, 6 radiological studies showed unilateral abnormalities but AVS indicated contralateral aldosterone secretion (fig. 4).

\section{Surgery and histopathological results (table 4)}

Sugery was proposed to patients with lateralised aldosterone secretion. In the case of discordant results between radiological studies and AVS results, the decision to operate or not was shared with the patient. Thirty patients had laparoscopic unilateral adrenalectomy, including 18 on the left side and 12 on the right

Table 2: Association between clinical determinants and bilateral AVS success.

\begin{tabular}{ll}
\hline & Success \\
\hline Age & $0.18(0.15)$ \\
\hline BMI & $0.36(0.0039)$ \\
\hline Office SBP & $0.54(0.0001)$ \\
\hline Office DBP & $0.17(0.42)$ \\
\hline Creatinine & $0.026(0.90)$ \\
\hline Potassium & $0.25(0.048)$ \\
\hline ARR & $0.22(0.080)$ \\
\hline
\end{tabular}

Data are Spearman's rank coefficient with associated $p$-value in parentheses. $\mathrm{BMI}=$ body mass index; $\mathrm{SBP}=$ systolic blood pressure; $\mathrm{DBP}=$ diastolic blood pressure; $\mathrm{ARR}=$ aldosterone renin ratio 
Table 3: Multivariate linear regression analysis of BMI with adrenal vein sampling success.

\begin{tabular}{lllll}
\hline Dependant variable & Predictor variables & Coefficient & (95\% confidence interval) & $p$-value \\
\hline Bilateral AVS success rate (\%) & BMI & 0.048 & $(0.016 ; 0.081)$ & 0.04 \\
\hline & Age & -0.0049 & $(-0.020 ; 0.010)$ & 0.52 \\
\hline & Sex & 0.14 & $(-0.27 ; 0.55)$ & $(-0.0057 ; 0.015)$ \\
\hline
\end{tabular}

Adjusted $\mathrm{R}^{2}=0.22$

Age, sex and creatinine were used as predictors. AVS = adrenal vein sampling; BMI = body mass index

Table 4: Number of laparoscopic adrenalectomies and histological findings.

\begin{tabular}{ll}
\hline Adrenalectomy $(\mathrm{n})$ & $48 \%(30 / 68)$ \\
\hline Right adrenalectomy & $40 \%(12 / 30)$ \\
\hline Left adrenalectomy & $60 \%(18 / 30)$ \\
\hline Histology reports $(\mathrm{n})$ & $30 / 30$ \\
\hline Adrenal adenoma & $74 \%$ \\
\hline Adrenal hyperplasia & $20 \%$ \\
\hline Normal adrenal & $7 \%$ \\
\hline
\end{tabular}

side. Most histopathological results showed adrenal adenoma $(22 / 28)$ followed by adrenal hyperplasia (6/28). Computed imaging was concordant with histopathological reports in $71 \%$ of the cases. Two adenoma revealed by histopathology were not detected by radiological studies.

\section{Discussion}

This study is the first to report that some clinical characteristics such as BMI, sex or creatinine may influence the success rate of AVS. This study also confirms previous reports showing the high rate of discordant results between radiological imaging such as CT scans or MRI with functional assessement of aldosterone secretion by AVS. Finally, in patients who had one of the adrenal glands removed, the results also confirmed that radiological findings show a relatively high number of discordant results.

Although several determinants of AVS success have been reported in the literature, it is surprising that patients characteristics have not been reported so far [11-13]. In the present study, male sex, a high BMI or a high plasma creatinine were significantly associated

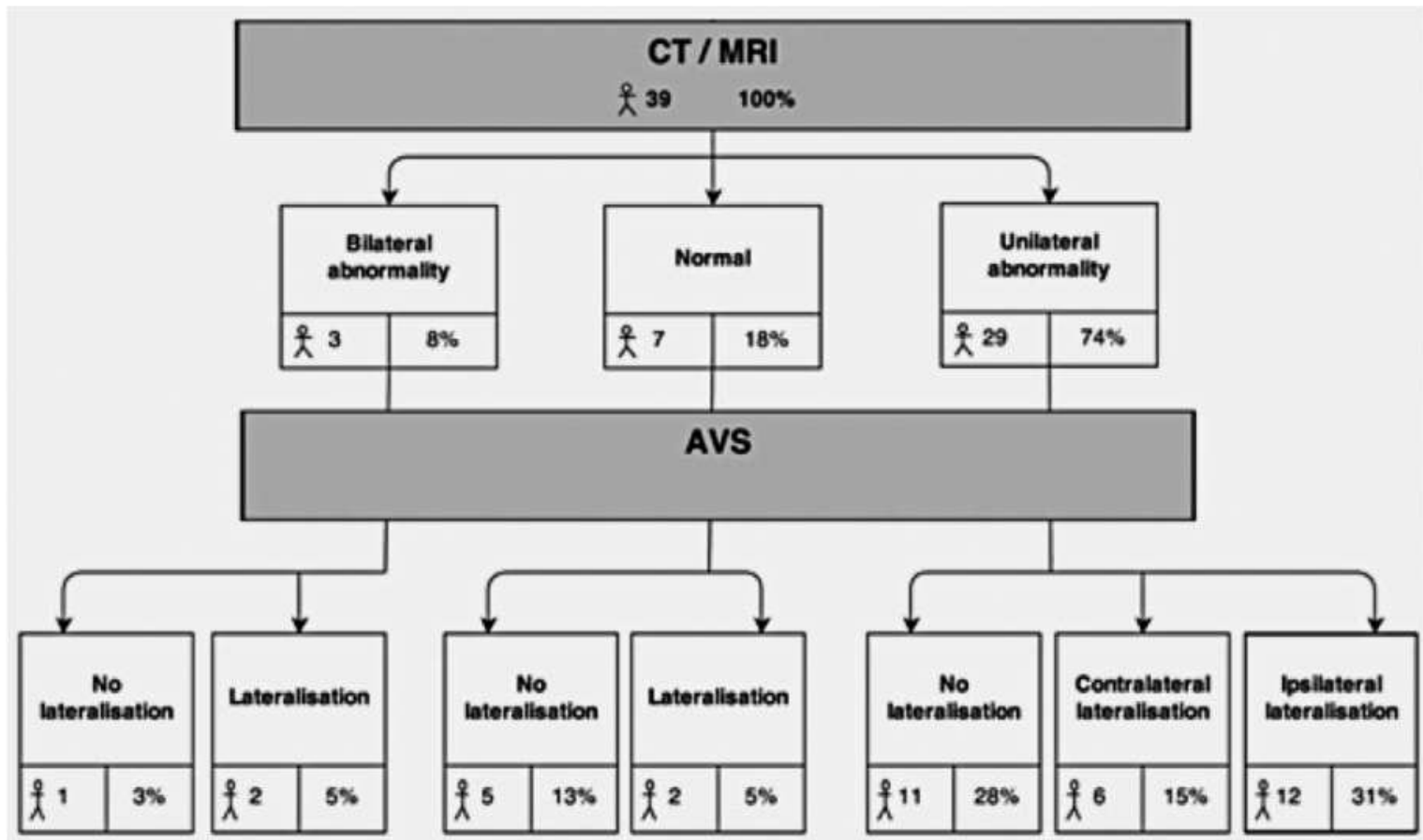

Figure 4: Comparison of radiological and AVS findings. AVS = adrenal vein sampling. 
with a greater success rate of having both adrenal veins catheterised. Only BMI persisted as a significant success factor in the multivariate analysis. This probably reflects the effect of sex on creatinine or BMI, which were both higher in men. The positive effect of BMI may be secondary to the increased intra-abdominal pressure found in obese patients [14]. Indeed, it has been shown that elevation in intra-abdominal pressure is associated with haemodynamic changes, such as renal venous pressure and decreased venous return, renal or visceral blood flow $[15,16]$. This increase in abdominal pressure may well have an impact on adrenal vein pressure and flow, which may increases adrenal vein diameter and facilitate catheterisation.

In our retrospective study, the overall success of bilateral catheterisation of adrenal veins was $60 \%$. In the literature, the rate of success ranges between $8 \%$ to $98 \%$, which largely depends on the implementation of a standardised operating procedure, the use of guiding CT during the procedure of adrenal vein sampling, the presence of an experienced interventional radiologist and a multidisciplinary approach [17]. Our success rate is in the range of the German Conn's registry after they implemented standardised procedures in order to increase the rate of success [17]. Locally, the presence of a single experienced interventional radiologist using a standardised protocol with the help of a CT scan to localise the adrenal veins may explain the similar success range. The cutoff value of the SI is logically very important in determining the success of AVS: the higher the cutoff, the lower the rate of success $[17,18]$. In the AVIS study, Rossi et al. showed that most of the major international referral centers are using different cutoffs to assess selectivity [19]. In 2014, the expert consensus statement on the use of AVS was published and clearly defined cutoff values which should be used for AVS with or without stimulated conditions [9]. The SI value for unstimulated AVS is recommended to be $\geq 2$, which we used as a referrence in our study.

In order to increase the AVS success rate, several proposals have been made, such as assessing cortisol during the AVS procedure (intra-procedural cortisol measurement, IPCM). A few studies showed that IPCM increases success rate and can avoid a second procedure in the event of bilateral AVS failure in the first place [20, 21]. Unfortunately, this method has some disadvantages. Indeed, direct cortisol assessment requires time (20-120 minutes) during which the patient is required to remain in the recovery room with a femoral shealths in situ, which may increase the risk of complications such as adrenal infarct, adrenal vein thrombosis, bleeding and infection. Some centres use a portable cortisol analyser that can be operated di- rectly in the radiology room, and thereby reduce the transport time to the laboratory. Unfortunately, the portable analysers are very expensive. The use of plasma metanephrine instead of cortisol to assess the selectivity of AVS is a new promising method which may replace cortisol in the future [22].

Finally, high-resolution CT can be used during the AVS procedure to provide visual and anatomical conformation of correct cannulation and thus reduces the risk of failure, particularly from the right adrenal vein [23].

\section{AVS and CT/MRI adrenal imaging discrepancies}

The distinction between unilateral or bilateral excess aldosterone secretion is essential to guide the choice of the most adequate treatment in patients with PA in whom surgery is an option. If imaging studies, such a CT scan or MRI have the advantage of availability, both may either miss aldosterone producing microadenomas resulting in inappropriate exclusion of surgery or detect nonfunctionning adrenal adenomas, which may lead to unnecessary adrenalectomy. Indeed, Kempers et al. published a systematic review based on more than 950 patients and 40 studies, which revealed that a discrepancy between AVS and adrenal imaging was present in $37.8 \%$ of the cases [24]. In our study, we found that $53 \%$ of adrenal imaging that did not match with the AVS results. Forty-three percent of these patients would therefore have been operated inappropriately, of whom 11 (28\%) showed a unilateral abnormality on adrenal imaging but no aldosterone lateralisation on AVS, 6 patients (15\%) showed lateralisation of secretion on AVS but on the opposite side compared with adrenal imaging and finally 4 patients (10\%) in whom adrenal imaging showed either an absence of abnormality or bilateral abnormality. However, CT and MRI remain important for the detection of anatomical variants before an adrenalectomy, in patients for whom PA is caused by a rare adrenal carcinoma or in patients whose health prohibits an AVS procedure. In these cases, adrenal imaging should still be considered a complementary technique.

Interestingly, the discordance between radiology and histopathology fell down to $29 \%$. This might reflect therapeutic decisions where adrenalectomy was proposed only if AVS and radiological images were concordant. The definite proof of AVS superiority over radiological studies as a decision tool as to whether to operate would have been obtained if follow-up data on hypertension control or cure in patients with discordant results (clear lateralisation of aldosterone secretion with no or controlateral adrenal nodule on radiological images) were available. 


\section{Limitations}

There are several limitations in our study. First, the study was retrospective, limiting the level of evidence. However, as reviewed recently, most of the published studies on this topic are retrospective [9]. As a result of the retrospective nature of the study, some clinical data were missing for some of patients, especially when patients were referred from other centres. Finally, AVS was performed without cosyntropin infusion, which is used for enhancing plasma cortisol concentrations, reducing stress-induced cortisol and aldosterone secretion during sequential AVS. However, there is no consensus so far on the use or non-use of cosyntropin infusion before or during AVS and some centers have abandoned cosyntropin infusion as it may increase the number of cases identified as bilateral $[9,10]$.

\section{Conclusion}

Clinical characteristics of patients with PA may influence the rate of success of AVS and may prepare the interventional radiologist for a difficult procedure. The use of a CT scan as a decision tool to perform unilateral adrenalectomy is not sufficient and biological assessement of aldosterone secretion lateralisation with AVS is essential in the work-up of patients who are candidates for adrenalectomy. Finally, follow-up studies to determine the rate of cure and control of hypertension are needed in order to define the use of AVS and radiological studies as guides to treatment in patients with primary aldosteronism.

\section{Disclosure statement}

National Fund: FN 32003B-149903. No other potential conflict of interest relevant to this article was reported.

\section{References}

1 Rossi GP G, Bernini C, Caliumi G, Desideri B, Fabris C, Ferri, et al. A prospective study of the prevalence of primary aldosteronism in 1,125 hypertensive patients. J Am Coll Cardiol. 2006;48(11):2293300.

2 Calhoun DA, Nishizaka MK, Zaman MA, Thakkar RB, Weissmann P. Hyperaldosteronism among black and white subjects with resistant hypertension. Hypertension. 2002;40(6):892-6.

3 Strauch B, Zelinka T, Hampf M, Bernhardt R, Widimsky Jr J. Prevalence of primary hyperaldosteronism in moderate to severe hypertension in the Central Europe region. J Hum Hypertens. 2003;17(5):349-52.

4 Savard S, Amar L, Plouin P-F, Steichen O. Cardiovascular Complications Associated With Primary Aldosteronism: A Controlled CrossSectional Study. Hypertension. 2013;62(2):331-6.

5 Sawka AM, Young WF, Thompson GB, Grant CS, Farley DR, Leibson C, et al. Primary aldosteronism: factors associated with normalization of blood pressure after surgery. Ann Intern Med. 2001;135(4): 258-61.
6 van der Linden P, Steichen O, Zinzindohoue F, Plouin PF. Blood pressure and medication changes following adrenalectomy for unilateral primary aldosteronism: a follow-up study. J Hypertens. 2012;30(4):761-9.

7 Rossi GP, Cesari M, Cuspidi C, Maiolino G, Cicala MV, Bisogni V, et al. Long-Term Control of Arterial Hypertension and Regression of Left Ventricular Hypertrophy With Treatment of Primary Aldosteronism. Hypertension. 2013.62(1):62-9.

8 Ishidoya S, Kaiho Y, Ito A, Morimoto R, Satoh F, Ito S, et al. Singlecenter outcome of laparoscopic unilateral adrenalectomy for patients with primary aldosteronism: lateralizing disease using results of adrenal venous sampling. Urology. 2011;78(1):68-73.

9 Rossi GP, Auchus RJ, Brown M, Lenders JW, Naruse M, Plouin PF, et al. An expert consensus statement on use of adrenal vein sampling for the subtyping of primary aldosteronism. Hypertension, 2014;63(1):151-60.

10 Daunt N. Adrenal vein sampling: how to make it quick, easy, and successful. Radiographics. 2005;25Suppl1:S143-58.

11 Harvey A, Pasieka JL, Kline G, So B. Modification of the protocol for selective adrenal venous sampling results in both a significant increase in the accuracy and necessity of the procedure in the management of patients with primary hyperaldosteronism. Surgery. 2012;152(4):643-9; discussion 649-51.

12 Mulatero PC, Bertello N, Sukor R, Gordon D, Rossato N, Daunt, et al. Impact of different diagnostic criteria during adrenal vein sampling on reproducibility of subtype diagnosis in patients with primary aldosteronism. Hypertension. 2010;55(3):667-73.

13 Young WF, Stanson AW. What are the keys to successful adrenal venous sampling (AVS) in patients with primary aldosteronism? Clin Endocrinol. (Oxf), 2009;70(1):14-7.

14 Lambert DM, Marceau S, Forse RA. Intra-abdominal pressure in the morbidly obese. Obes Surg. 2005;15(9):1225-32.

15 Sugerman HJ. Effects of increased intra-abdominal pressure in severe obesity. Surg Clin North Am. 2001;81(5):1063-75, vi.

16 Barnes GE, Laine GA, Giam PY, Smith EE, Granger HJ. Cardiovascular responses to elevation of intra-abdominal hydrostatic pressure. Am J Physiol. 1985;248(2Pt2):R208-13.

17 Vonend ON, Ockenfels X, Gao B, Allolio K, Lang K, Mai, et al. Adrenal venous sampling: evaluation of the German Conn's registry. Hypertension. 2011;57(5):990-5.

18 Lethielleux G, Amar L, Raynaud A, Plouin PF, Steichen O. Influence of diagnostic criteria on the interpretation of adrenal vein sampling. Hypertension. 2015;65(4):849-54.

19 Rossi GP, Barisa M, Allolio B, Auchus RJ, Amar L, Cohen D, et al. The Adrenal Vein Sampling International Study (AVIS) for identifying the major subtypes of primary aldosteronism. J Clin Endocrinol Metab. 2012;97(5):1606-14.

20 Reardon MA, Angle JF, Abi-Jaoudeh N, Bruns DE, Haverstick DM, Matsumoto AH, et al. Intraprocedural cortisol levels in the evaluation of proper catheter placement in adrenal venous sampling. J Vasc Interv Radiol. 2011;22(11):1575-80.

21 Rossi E, Regolisti G, Perazzoli F, Negro A, Grasselli C, Santi R, et al. Intraprocedural cortisol measurement increases adrenal vein sampling success rate in primary aldosteronism. Am J Hypertens. 2011;24(12):1280-5.

22 Dekkers T, Deinum J, Schultzekool LJ, Blondin D, Vonend O, Hermus AR, et al. Plasma metanephrine for assessing the selectivity of adrenal venous sampling. Hypertension. 2013;62(6):1152-7.

23 Onozawa S, Murata S, Tajima H, Yamaguchi H, Mine T, Ishizaki A, et al. Evaluation of right adrenal vein cannulation by computed tomography angiography in 140 consecutive patients undergoing adrenal venous sampling. Eur J Endocrinol. 2014;170(4):601-8.

24 Kempers MJ, Lenders JW, van Outheusden L, van der Wilt GJ, Schultze Kool LJ, Hermus AR, et al. Systematic review: diagnostic procedures to differentiate unilateral from bilateral adrenal abnormality in primary aldosteronism. Ann Intern Med. 2009;151(5):329-37. 